\title{
IoV-based Deployment and Scheduling of Charging Infrastructure in Intelligent Transportation Systems
}

\author{
W. Ejaz, M. Naeem, S. K. Sharma, A. M. Khattak, M. R. Ramzan, A. Ali, and A. Anpalagan
}

\begin{abstract}
Internet of vehicles (IoV) is an emerging paradigm to exchange and analyze information collected from sensors using wireless technologies between vehicles and people, vehicles and infrastructure, and vehicles-to-vehicles. With the recent increase in the number of electric vehicles (EVs), the seamless integration of IoV in EVs and charging infrastructure can offer environmentally sustainable and budget-friendly transportation. In this paper, we propose an IoV-based framework that consists of deployment and scheduling of a mobile charging infrastructure. For the deployment, we formulate an optimization problem to minimize the total cost of mobile charging infrastructure placement while considering constraints on the number of EVs that can be charged simultaneously. The formulated problem is mixedinteger programming and solved by using the branch and bound algorithm. We then propose an IoV-based scheduling scheme for EVs charging to minimize travel distance and charging costs while satisfying the constraints of charging time requirement of EVs and resources of a charging station. We consider passive road sensors and traffic sensors in the proposed IoV-based scheduling scheme to enable EV users for finding a charging station that can fulfill their requirements, as well as to enable service providers to know about the demand in the area. Simulation results illustrate the significant impact of the optimal deployment of charging infrastructure and scheduling optimization on the efficiency of EVs charging.
\end{abstract}

Index Terms-Charging infrastructure, electric vehicles, Internet of vehicles, scheduling optimization.

\section{INTRODUCTION}

Recently, there is a significant increase in the number of electric vehicles (EVs) to make cities greener with energyefficient and low/zero-carbon transportation system [1]. The charging demand is growing with the increase in the number of EVs, which needs efficient deployment and scheduling of a mobile charging infrastructure. To enable the faster adoption of EV technology, it is very crucial to deploy efficient public

Corresponding authors: Waleed Ejaz and Muhammad Naeem

W. Ejaz is with the Department of Engineering \& Applied Science, Thompson Rivers University, Kamloops, BC, Canada (e-mail: waleed.ejaz@ieee.org).

M. Naeem is with the COMSATS University Islamabad, Wah Campus, Wah Cantonment 47040, Pakistan (e-mail: muhammadnaeem@gmail.com).

S. K. Sharma is with SnT, University of Luxembourg, Luxembourg (e-mail: shree.sharma@ieee.org).

A. M. Khattak is with the College of Technological Innovation, Zayed University, 144534, UAE (e-mail: asad.khattak@zu.ac.ae).

M. Rashid is with the COMSATS University Islamabad, Wah Campus, Wah Cantonment 47040, Pakistan (e-mail:rashidramzan33@yahoo.com).

A. Ali is with the COMSATS Institute of Information Technology, Lahore, Pakistan (e-mail: amjad.ali@ @iitlahore.edu.pk).

A. Anpalagan is with the Department of Electrical and Computer Engineering, Ryerson University, Toronto, ON, Canada (email: alagan@ee.ryerson.ca).

We acknowledge the support of the Natural Sciences and Engineering Research Council of Canada (NSERC). This research work was supported in part by Zayed University Policy Research Incentive Fund. charging infrastructure by considering various aspects such as charging time and travel time for the EVs. However, higher capital costs of deploying charging stations and uneven power demands could be problematic, leading to the need for understanding the relationship among customer service quality, peak demand power, and port utilization. Moreover, one crucial issue for the EV charging stations, which usually have different charging modes to meet several requirements of the EVs, is to utilize the charging capacity effectively to lower the service dropping rate. Therefore, it is crucial to efficiently manage the charging demands of EVs and infrastructure while considering the heterogeneous charging requirements of EVs. In this context, Internet of vehicles (IoV) is an emerging paradigm that can enable EVs and mobile charging infrastructure to use sensing and communication technologies for sustainable and budget-friendly intelligent transportation system as well as effectively manage consumer resources and reduce operating cost [2]-[4]. The main components of an IoVbased charging management system include sensing devices, actuators, communications technologies, and servers for data processing. The IoV-based charging management framework can handle various functions, including the deployment of mobile charging infrastructure, scheduling, and enabling a smart and automated billing system. Furthermore, automatic charging request generation from the sensors in the EVs, and the subsequent broadcasting of information from a grid server to the EVs can significantly help energy providers to acquire energy demand information in advance and to maximize the revenue [5]. Moreover, EVs can be effectively charged via mobile charging stations while considering their dynamicity of energy demand [6]. Also, it is essential to provide an optimal charging solution to the EV users to fulfill their charging demand with the given budget constraint. Therefore, IoV-based mobile charging infrastructure deployment and scheduling is essential for the success of future intelligent transportation systems.

In this direction, a comprehensive survey of the existing international standards and charging modes for EVs is provided in [7]. The latest developments and challenges in the realization of EVs charging systems are also highlighted. EVs can then locally or local servers can get the following information by using sensing and communication technologies from the mobile charging infrastructure: the charging station location, the available number of plugs, the average wait time to get a plug, the average time for charging, and the cost to be paid for charging. Also, EVs or local servers can get information about traffic congestion on the roads by using global positioning system. Using this information, EVs 
locally or local servers can find optimal charging stations based on user requirements in terms of charging, time, budget, etc. Thus, we need updated information from the EVs and charging stations to optimize the selection of a charging station according to user requirements. On the other hand, the EVs fleet operator or aggregators need real-time parameters from the EVs for vehicle-to-grid support. These parameters include the remaining EVs charge, capacity of charging, as well as driving patterns. This information can significantly enhance the demand side management and other ancillary services.

IoV-based framework for charging management can be categorized into the deployment of mobile charging infrastructure and scheduling optimization of EVs charging:

- Deployment of mobile charging infrastructure: Lack of sufficient charging stations to support the increasing number of EVs is one of the crucial issues to be addressed. Also, due to the relatively long charging process for the EVs, introducing charging stations in the limited space of the existing gas stations is not a viable solution. In addition, selecting appropriate location for mobile charging station deployment is a challenging issue because of its expensive deployment process [8]. Another issue in EV charging is the unbalanced supply and demand since there might be many stations idle in the remote areas and not enough stations in the areas with higher requirements. This requires mobile charging infrastructure in the appropriate areas while taking account of different factors such as mileage, demand priority, and the distribution of customers. Therefore, it is important to deploy charging stations with the objectives of maximizing the coverage area and minimizing the total cost required for the deployment of charging infrastructure.

- Scheduling optimization of EVs charging: To deal with the scheduling of a charging load from a large number of EVs, an IoV-based interactive and efficient charging management needs to be investigated [9]. The IoV-based scheduling can enable the real-time interactions among the charging stations and the EVs by utilizing various devices and sensors. The scheduling of EVs charging can be optimized by considering different objectives, such as maximizing the total profit of a service provider and minimizing the service price from the perspective of $\mathrm{EV}$ users. Furthermore, several parameters, including wait time, total charging time, and distance between EVs and charging stations, should be taken into account while performing the scheduling optimization.

\section{A. Contributions}

In this paper, we propose an IoV-based framework for deployment mobile charging infrastructure deployment and scheduling for intelligent transportation systems. The main contributions of this paper are summarized below:

- We propose an optimal deployment mechanism to minimize deployment cost of mobile charging infrastructure while considering charging requirements of EVs.

- We propose a scheduling scheme for optimizing charging schedule of EVs with joint minimization of travelling cost for changing and charging cost in terms of time and charging price.

- The performance gains achieved in both the cases are depicted via simulation results and some promising future research directions are provided.

The rest of the paper is organized as follows: Section II provides an overview of related work. System model is presented in Section III. We present the proposed IoV-based framework which consists of optimal deployment of mobile charging stations and scheduling optimization of EVs in Section IV. Performance evaluation and simulation results are discussed in the Section V. Finally, Section VI draws conclusions and outlines future research directions.

\section{RELATED WORKS}

In this section, we provide an overview of the state-of-theart sensors and communication technologies assisted (i) deployment of mobile charging infrastructure and (ii) scheduling optimization of EVs charging.

\section{A. Deployment of Mobile Charging Infrastructure}

Regarding the deployment of charging infrastructure, distribution companies are interested in reducing the planning and deployment cost while maximizing profit. On the other hand, EV users strive to increase their benefits by finding optimal charging station which can fulfill their requirements. The total cost of EV batteries, charging stations, energy storage systems, and electricity demand charges is minimized by optimal deployment of charging stations in [8]. The formulated problem is a mixed integer linear programming model for which results are demonstrated in real-world scenario of bus network in Salt Lake City, Utah. A two-stage scheme is proposed for simultaneous allocation of EV charging stations and distributed renewable sources is proposed in [10]. In the first step, candidate locations are selected based on reliability index, attraction index, and price of land index. Whereas in the second step, optimum number of charging stations and probabilistic model for charging stations placement is determined based on the given specifications. Authors in [11] investigated optimal deployment of charging stations to maximize the covered path flows while considering path deviation and non-linear elastic demand. A tailored branch and pricing method is proposed to solve the optimal deployment problem. A joint deployment of charging stations and photovoltaic power plants for EVs is studied in [12]. The EV routing and charging problem is solved by label-setting algorithm which considers time-dependent charging fee for different charging stations. Authors then adopted a surrogate-based optimization algorithm optimally design charging stations, photovoltaic plants, and time-dependent charging fee. A heuristic method is proposed for optimal deployment of EV charging stations is proposed in [13] to minimize the charging time while considering constraints on driving range and traffic conditions.

Furthermore, authors in [14] proposed an EV charging station placement method by considering an integrated electrical and transportation network, and the costs related to the voltage regulation, EV station construction, protection device update, 
and distribution expansion. Subsequently, the authors analyzed the impact of protection and voltage regulation upgrades on the placement of EV charging stations and showed the significance of incorporating protection device upgrade while planning EV charging stations in urban areas. Authors in [15] developed non-deterministic and agent-based models to minimize the total EV travel distance and to find the optimized locations of EV charging stations, respectively. Binary lightning search algorithm is adopted for optimal sizing and location of fastcharging stations in [16]. Authors considered different aspects such as grid power loss, transportation loss, and build-up costs while utilizing road traffic density, battery state of charge and Google maps. Authors in [17] investigated optimization model for the optimal placement of charging station to minimize cost and ease of use. The potential locations are determined and mixed integer linear programming problem is solved to obtain optimal solution for the placement of charging stations.

\section{B. Scheduling Optimization of EVs Charging}

The scheduling of EV aggregator in the uncertain environment can reduce the cost and improving the charging of EVs [18]. An interval optimization approach is proposed to model uncertain grid price to solve a multi-objective problem which considers minimization of deviation profit and maximization of average profit. An optimization model is presented in [19] to minimize the annual total scheduling costs. A heuristic procedure is proposed for optimal solution while considering recharging trips and heterogeneous EVs. The authors in [20] proposed a predictive algorithm for the low-complexity scheduling of EVs charging in which non-causal information about the future arrivals of EVs is not available but only the estimated statistical information. A distributed algorithm to jointly optimize the routing selection and charging schedule of IoV-based EV networks is proposed in [21]. This joint optimization problem is formulated as mixed-integer nonlinear programming, and this NP-had problem is tackled by using an approximate distributed algorithm, which enables to calculate a routing and charging solution in a distributed way by the EV users and a system operator. The authors in [22] developed a fast-charging station model to generate the statistics of charging and queuing of the EV charging stations for any provided customer/vehicle data. Via the analysis and numerical results, it has been shown that increasing the number of ports for a given charging station increases the customer service quality by decreasing the wait time, however, on the other hand, reduces port utilization. A dynamic wireless charging of EVs on the move and intelligent routing of EVs to enhance driving range is investigated in [23]. The first approach enables the exchange of power between the vehicle and the grid when the EV is moving while the second approach enables the EVs to utilize the mobile energy disseminators (MEDs), i.e., mobile charging stations deployed in buses, trucks and heavy goods vehicles. A graph-based shortest path algorithm is proposed for the intelligent routing of the EVs by using either MEDs or the static stations to minimize the overall travel time.

Authors in [24] investigated deterministic based scheduling of EVs aggregator while considering grid price uncertainty.
The upstream grid price uncertainty is modeled using robust optimization technique. The results of proposed scheduling scheme can be used to develop charging and discharging strategies by the operators. An optimal scheduling scheme for EVs and photo-voltaic systems is proposed in [25] for residential complexes while considering real-time pricing mechanism. The optimal operation of energy systems in residential complexes is modeled as mixed-integer linear programming and a general algebraic modeling system is adopted to present simulation results in different scenarios. The relationship between the EV selection and service dropping rate of a charging station is investigated in [26]. An optimal pricing method is formulated to coordinate the charging of EVs, and subsequently to minimize the EV charging station's service dropping rate. Also, authors in [27] proposed a machine learningbased demand response and charging strategy to determine the charging time of the EVs while considering real-time pricing to minimize the overall energy cost. A Markov decision process is employed to model the charging control process, and the problem is then addressed by using dynamic programming and several machine learning algorithms, including shallow neural network, deep neural network, and k-nearest neighbors. Swappable batteries are also being considered as alternative EV recharging methods in EVs [28] which provides fast energy recharging service to the EV users and also a high degree of flexibility to the grid operators to perform load balancing. The problem of scheduling the battery charging station is studied to schedule the charging process of the underlying charging bays. The problem is formulated as a mixed-integer program and then is solved by utilizing a generalized benders decomposition algorithm.

A summary of the existing related work is given in Table I. Unlike the existing work, we propose an IoV-based framework that consists of deployment and scheduling of charging infrastructure management system while considering the minimization of installation cost, charging requirements of EVs, finding optimal charging station based on EV requirements. The proposed framework will also enable service providers to predict future demands of charging in a given area.

\section{System ModeL}

We consider a large city divided into small regions in which charging requests are handled by local servers in each partition. This is a valid assumption as the EVs will be charged by charging stations in the corresponding regions. This makes our proposed system semi-centralized which is scalable. Fig. 1 illustrates one region in a large city for the proposed IoV-based framework for deployment and scheduling of mobile charging infrastructure in intelligent transportation system. We consider $N_{P}$ number of possible authorized locations by regulatory authorities, where we can install the charging stations. The estimated number of connectors and estimated cost for the $m$-th potential location are denoted by $Y_{m}$ and $Z_{m}$, respectively. The number of connectors required to install at each charging station is based on the flow of EVs through a charging station. It is assumed that the each charging station can share the following information with the 
TABLE I

SuMmary OF RELATED WORKS [D: DePLOYMENT, S: SCHEDULing].

\begin{tabular}{|c|c|c|c|c|}
\hline Ref. & Objective & $\mathbf{D}$ & $\mathbf{S}$ & Solution \\
\hline$[8]$ & $\begin{array}{l}\text { The total cost is minimized by optimal } \\
\text { deployment of charging stations }\end{array}$ & $\checkmark$ & $x$ & $\begin{array}{l}\text { Results are demonstrated for a bus network in } \\
\text { Salt Lake City, Utah. }\end{array}$ \\
\hline$[10]$ & $\begin{array}{l}\text { Simultaneous allocation of charging stations } \\
\text { and distributed renewable resources }\end{array}$ & $\checkmark$ & $x$ & Two-stage scheme is proposed. \\
\hline$[11]$ & $\begin{array}{l}\text { Optimal deployment of charging stations to } \\
\text { maximize the covered path flows }\end{array}$ & $\checkmark$ & $x$ & $\begin{array}{l}\text { A tailored branch and pricing method is } \\
\text { proposed. }\end{array}$ \\
\hline$[12]$ & $\begin{array}{l}\text { Joint deployment of charging stations and } \\
\text { photovoltaic power plants }\end{array}$ & $\checkmark$ & $x$ & $\begin{array}{l}\text { Label-setting algorithm and surrogate-based } \\
\text { optimization algorithm are proposed. }\end{array}$ \\
\hline$[13]$ & $\begin{array}{l}\text { Charging time minimization considering } \\
\text { driving range and traffic conditions }\end{array}$ & $\checkmark$ & $x$ & A heuristic method is proposed. \\
\hline$[14]$ & $\begin{array}{l}\text { Placement considering integrated electrical and } \\
\text { transportation network, and deployment costs }\end{array}$ & $\checkmark$ & $x$ & $\begin{array}{l}\text { Analyzed impact of protection and voltage } \\
\text { regulation on placement in urban areas. }\end{array}$ \\
\hline$[15]$ & $\begin{array}{l}\text { Minimize total EV travel distance and to find } \\
\text { the optimized locations of charging stations }\end{array}$ & $\checkmark$ & $\checkmark$ & $\begin{array}{l}\text { Developed non-deterministic and agent-based } \\
\text { models. }\end{array}$ \\
\hline$[16]$ & Optimal location and size of charging stations & $\checkmark$ & $x$ & Binary lightning search algorithm is adopted. \\
\hline$[17]$ & $\begin{array}{l}\text { The optimal placement of charging station to } \\
\text { minimize cost and ease of use }\end{array}$ & $\checkmark$ & $x$ & $\begin{array}{l}\text { Mixed integer linear programming problem is } \\
\text { solved to obtain optimal solution. }\end{array}$ \\
\hline$[18]$ & $\begin{array}{l}\text { Maximize the average profit and minimize the } \\
\text { deviation profit }\end{array}$ & $x$ & $\checkmark$ & An interval optimization approach is proposed \\
\hline$[19]$ & Minimize the annual total scheduling costs & $x$ & $\checkmark$ & Heuristic procedure is proposed. \\
\hline$[20]$ & Low-complexity scheduling of EVs charging & $x$ & $\checkmark$ & $\begin{array}{l}\text { A predictive algorithm is proposed to estimate } \\
\text { future arrival of EVs. }\end{array}$ \\
\hline$[21]$ & $\begin{array}{l}\text { Jointly optimize the routing selection and } \\
\text { charging schedule of IoV-based EV networks }\end{array}$ & $x$ & $\checkmark$ & $\begin{array}{l}\text { Approximate distributed algorithm is used to } \\
\text { solve the optimization problem. }\end{array}$ \\
\hline$[22]$ & $\begin{array}{l}\text { Generate the statistics of charging and } \\
\text { queuing of the EV charging stations }\end{array}$ & $x$ & $\checkmark$ & Developed a fast-charging station model. \\
\hline$[23]$ & $\begin{array}{l}\text { Dynamic wireless charging and intelligent } \\
\text { routing of EVs to enhance driving range }\end{array}$ & $x$ & $\checkmark$ & $\begin{array}{l}\text { A graph-based shortest path algorithm is } \\
\text { proposed. }\end{array}$ \\
\hline$[24]$ & $\begin{array}{l}\text { Deterministic scheduling of EVs aggregator } \\
\text { while considering grid price uncertainty }\end{array}$ & $x$ & $\checkmark$ & Robust optimization technique is proposed. \\
\hline$[25]$ & $\begin{array}{l}\text { Optimal operation of energy systems in } \\
\text { residential complexes }\end{array}$ & $x$ & $\checkmark$ & $\begin{array}{l}\text { A general algebraic modeling system is } \\
\text { adopted to obtain optimal solution. }\end{array}$ \\
\hline$[26]$ & $\begin{array}{l}\text { minimize the EV charging station's service } \\
\text { dropping rate }\end{array}$ & $x$ & $\checkmark$ & An optimal pricing method is proposed. \\
\hline [27] & $\begin{array}{l}\text { Determine the charging time of the EVs to } \\
\text { minimize the overall energy cost }\end{array}$ & $x$ & $\checkmark$ & $\begin{array}{l}\text { Dynamic programming and machine learning } \\
\text { algorithms are adopted for optimal solution. }\end{array}$ \\
\hline$[28]$ & $\begin{array}{l}\text { Schedule the charging process of the } \\
\text { underlying charging bays }\end{array}$ & $x$ & $\checkmark$ & $\begin{array}{l}\text { A generalized benders decomposition } \\
\text { algorithm is adopted for scheduling. }\end{array}$ \\
\hline
\end{tabular}

server on request: charging station location, available number of plugs, the average wait time to get a plug, the average time for charging, and the cost of charging. We consider $N_{V}$ number of EVs that are equipped with heterogeneous sensors to collect their location information, remaining battery life, etc. We assume that EVs and charging stations are equipped with communication technologies for both long-range (LTE/LTEA) and limited-range (WiFi) communication technologies and can communicate with the edge computing devices on the road sides or with the local servers. We further assume that the data exchanged between EVs, servers, and charging stations is encrypted.

There are different levels of charging defined in the litera- ture, depending on the charging time and power. For example, level 1 charging also called slow charging, where the charging is done by applying $120 \mathrm{VAC} / 16 \mathrm{~A}$ for $1.92 \mathrm{~kW}$ charging. The time it takes to charge a vehicle battery to its full capacity is about 10 hours [29]. Level 1 charging is usually done in houses or offices, where the EVs are generally parked for a long time. In level 2 charging also called standard charging, the charging is done by applying $208 \mathrm{~V}-240 \mathrm{VAC}, 12 \mathrm{~A}-80 \mathrm{~A}$ for the $2.5 \mathrm{~kW}-19.2 \mathrm{~kW}$ charging. It takes $6-8$ hours to charge an empty battery to its full capacity. Level 2 charging stations can be deployed in an area where EV users stay for a long time, e.g., offices. In both level 1 and level 2, the charging is done using the on-board battery charger. In level 3 charging, 


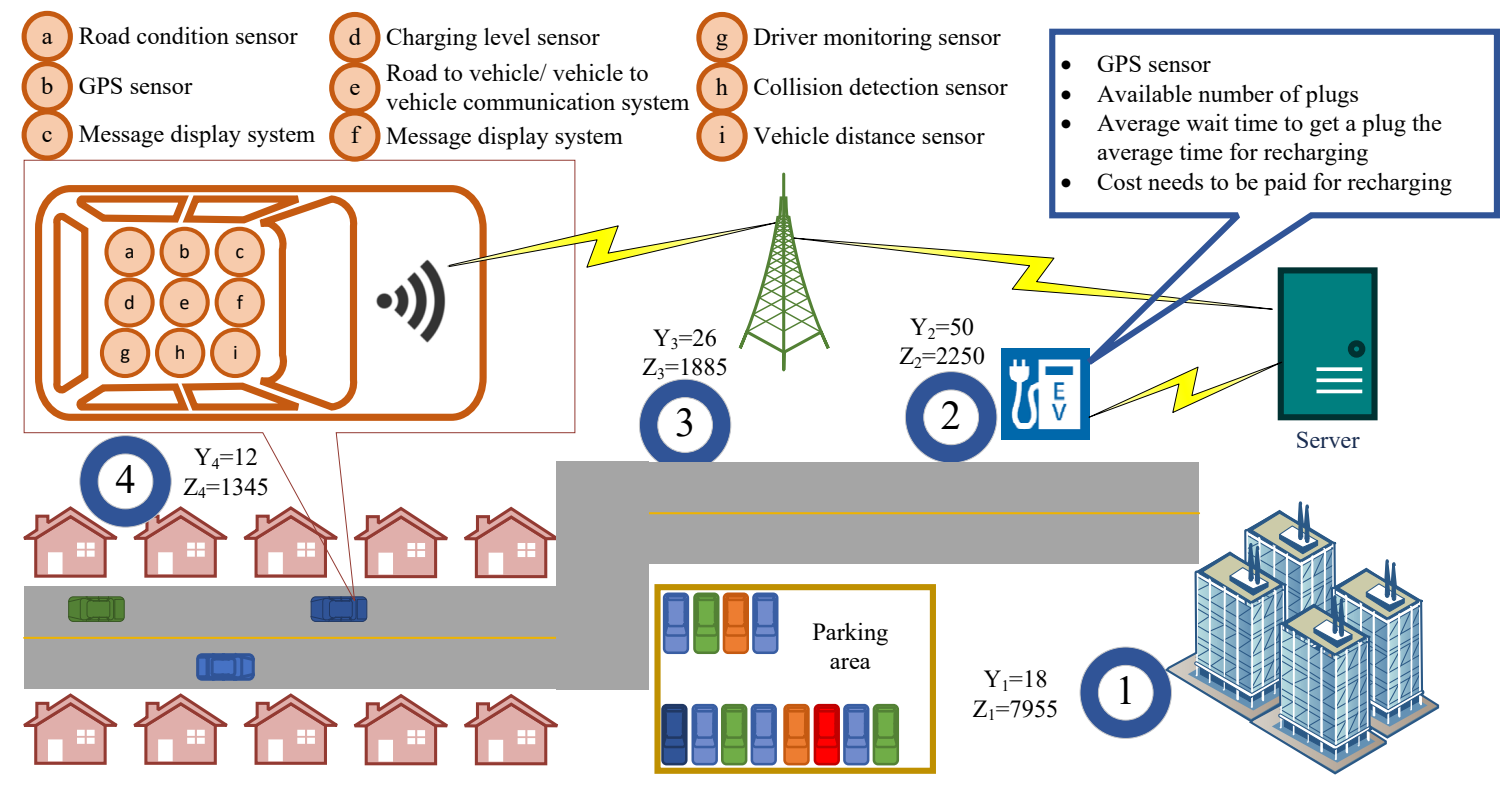

Fig. 1. System model for IoV-based mobile charging infrastructure deployment and scheduling in intelligent transportation system.

also called fast charging, the charging is done with up to $400 \mathrm{~A}$ for $240 \mathrm{~kW}$ charging power using an off-board charger. It takes approximately 30 minutes to charge a vehicle battery in this case. Level 3 charging is suitable in areas where the EV user is staying for a short time, e.g., shopping mall. The EV charging level characteristics for both AC and DC types according to the society of automotive engineers are given in Table II. Therefore, each charging station can offer different charging levels with varying cost. Let $G$ be the set of different charging levels available for the EVs. Each charging level $g \in G$ has a different associated cost for charging and time to charge. Without loss of generality and for illustration purposes, we consider a limited set of charging levels, i.e., $G=\{$ slow, normal, fast, boosted $\}$. The cost of slow charging for the EVs is very low; however, it takes more time to charge. On the contrary, the cost for boosted charging is very high and it takes less time for charging. We consider $C_{S}, C_{N}, C_{F}$, and $C_{B}$ as charging cost and $T_{S}, T_{N}, T_{F}$, and $T_{B}$ as charging time for slow, normal, fast and boosted charging, respectively, where $C_{S}<C_{N}<C_{F}<C_{B}$ and $T_{S}>T_{N}>T_{F}>T_{B}$.

\section{IOV-BASED FrAMEWORK FOR CHARGING MANAGEMENT}

The IoV-based framework for charging management consists of two main components: i) optimal placement of charging stations and ii) scheduling optimization of EVs charging.

\section{A. Placement of Charging Stations}

We propose a strategy for optimal placement of charging stations to minimize the installation cost. The local server in IoV-based charging management system is in-charge of all operations and have complete knowledge of EV user requirements and their density in a given area. Thus, the local server is responsible for the placement of charging stations. We consider both static and mobile charging stations placement.
Therefore, mobile charging station placement can be an ongoing task based on the EV users requirements. The problem of a charging station placement can be formulated with different objectives, e.g., maximizing coverage, minimizing cost, etc. It is assumed that the charging station at $m$-th $\in N_{P}$ potential location offers $Y_{m}$ number of estimated connectors and $Z_{m}$ amount of deployment cost. The number of connectors $Y_{m}$ for the $m$-th potential location depends on the flow of EVs through it. We assume a known and fixed number of connectors at each charging station. We propose a solution to find optimal locations for charging stations placement out of possible $N_{P}$ potential locations. We can have a decision variable $X_{m}$ :

$$
X_{m}= \begin{cases}1, & \text { if charging station is placed at } m \text {-th location, } \\ 0, & \text { otherwise }\end{cases}
$$

Then, the total cost of placement of charging stations can be written as: $\sum_{m=1}^{N_{P}} Z_{m} X_{m}$. The placement cost of charging station at the $m$-th location can be represented as $Z_{m}=$ $\left(A C_{L}+C_{C}\right) Y_{m}+C_{\text {init }, m}$, where $A$ is the land area requirement for one vehicle to recharge (we consider $\mathrm{A}=25 \mathrm{ft}^{2}$ ), $C_{L}$ is the rental cost of land per $f t^{2}, C_{C}$ is cost per connector. The cost of connector is directly proportional with the power rating of the connector. The initial cost (cost for basic structure and construction) of a charging station is denoted by $C_{\text {init }, m}$. The electrification cost of a charging station is not considered separately as we consider it as a part of $C_{\text {init }, m}$. We formulate an optimization optimization problem to minimize the total cost of placement of charging stations while considering the cost of deployment and connectors for each charging station: 
TABLE II

EV CHARGING LEVEL CHARACTERISTICS [30].

\begin{tabular}{lllllcl}
\hline $\begin{array}{l}\text { Power } \\
\text { level }\end{array}$ & Type & $\begin{array}{l}\text { Voltage level } \\
{[\mathbf{V}]}\end{array}$ & $\begin{array}{l}\text { Capacity } \\
{[\mathbf{A}]}\end{array}$ & $\begin{array}{l}\text { Power } \\
\text { capacity } \\
{[\mathbf{k W}]}\end{array}$ & $\begin{array}{l}\text { Charger } \\
\text { type [kW] }\end{array}$ & Charging time \\
\hline Level 1 & AC & 120 VAC & 16 & 1.92 & - & PHEV:7 h/BEV: 10 h \\
Level 2 & AC & 240 VAC & 80 & 19.2 & 7 & PHEV:3 h/BEV: 7 h \\
Level 3 & AC & - & $400 \mathrm{~A}$ & $>20$ & - & Under development \\
Level 1 & DC & 200-500 VDC & $<80$ & Up to 40 & 20 & PHEV:22 min/BEV: $1.2 \mathrm{~h}$ \\
Level 2 & DC & 200-500 VDC & $<200$ & Up to 100 & 45 & PHEV:10 min/BEV: 20 min \\
Level 3 & DC & 200-600 VDC & $<400$ & Up to 240 & - & Under development \\
\hline
\end{tabular}

$$
\begin{gathered}
\min _{X_{m}}: \sum_{m=1}^{N_{P}} Z_{m} X_{m} \\
\text { Subject to }: \sum_{m}^{N_{P}} X_{m} Y_{m} \geq \beta N_{V} \\
X_{m} \in\{0,1\} .
\end{gathered}
$$

where constraint ensures that the total number of connectors installed in all the charging stations must be sufficient to satisfy the charging requirements of a minimum $\beta \%$ of EVs in that area. In other words, $\beta \%$ of EVs in the serving area must be able to charge simultaneously.

The problem in (2) is mixed-integer programming, which is generally NP-hard. The optimal solution can be obtained by enumerating all possible combinations of $X_{m}$. However, this is a computationally expensive solution and not suitable for a large number of potential locations and the number of charging stations. To tackle this issue, we adopted branch and bound algorithm (See [31] for more details) for the placement of charging stations given in Algorithm 1. The computational complexity of the branch and bound algorithm in the average case is notably low, which can be considered as a reasonable estimate of the performance [31].

\section{B. Scheduling Optimization of EVs Charging}

Fig. 2 presents a flow diagram for the proposed scheduling optimization depicting the frame exchange among the EVs, local server, and charging stations. In our proposed scheduling optimization scheme, the EVs can request for charging to local server by sending charging request packet if the residual charging is less than a pre-set threshold. The residual energy will be determined by the sensors installed on the EVs. The threshold can be setup considering the EVs have sufficient charging to reach charging stations. The request packet contains the requesting EVs ID, location, and budget. For efficient charging request, we adopt RF-MAC protocol proposed in [32], which ensures that the EVs with residual charging less than threshold will get channel access before giving access to any other EV or devices. Similar to RF-MAC protocol, the EVs other than requesting for charging and devices are forced to freeze their data transmission to give priority to the EVs with residual charging less than threshold. The local server will receive
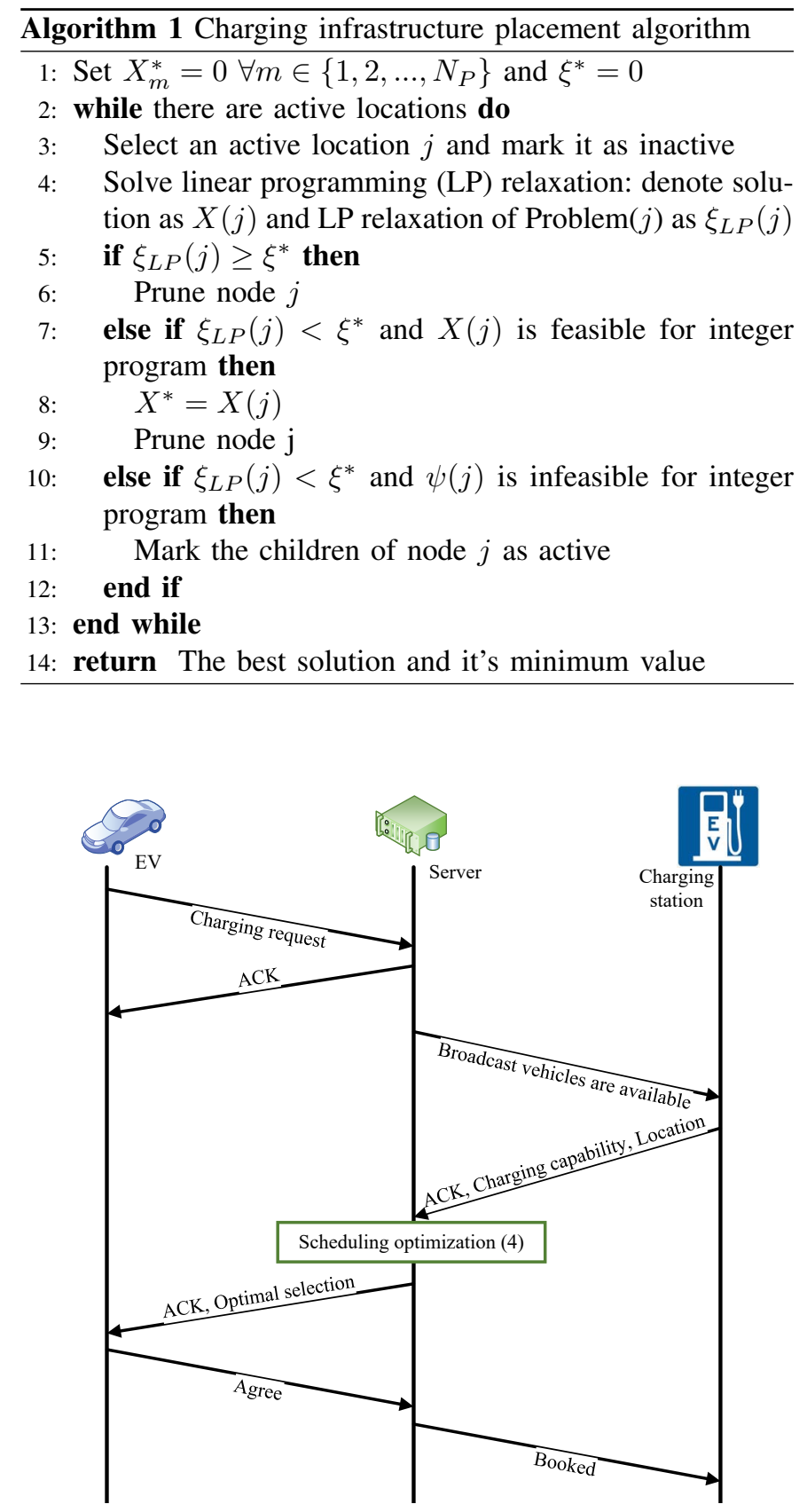

Fig. 2. Flow diagram for the scheduling optimization of EVs. 
charging request packet and sends an acknowledgement to the EVs and broadcast request to the charging stations.

We consider that charging stations can be mobile or static. The charging stations willing to participate in the bid then sends acknowledgment to the server with their location, available number of plugs, the average wait time to get a plug, the average time for recharging, and cost needs to be paid for charging. The server will also get information about traffic congestion on the road to determine travel time to each charging station. The server then performs optimization based on the information from EVs and charging stations as well as traffic congestion. The objective is to minimize the utility function, which consists of the distance between a charging station and the EV, travel time EV takes to reach charging station, the charging price, and wait time.

We consider that EVs can charge from charging stations deployed at $N_{P}$ potential locations. Then the utilization matrix can be written as:

$$
U=\left[\begin{array}{ccccc}
u_{11} & u_{12} & u_{13} & \ldots & u_{1 N_{P}} \\
u_{21} & u_{22} & u_{23} & \ldots & u_{2 N_{P}} \\
\vdots & \vdots & \vdots & \vdots & \vdots \\
u_{N_{V} 1} & u_{N_{V} 2} & u_{N_{V} 3} & \ldots & u_{N_{V} N_{P}}
\end{array}\right]
$$

where $u_{n m} \in\{0,1\}$ is 1 when $n$-th $\mathrm{EV}$ is using charging station deployed at $m$-th location and 0 otherwise.

We also define the travel distance for all EVs to potential locations for the charging stations:

$$
D=\left[\begin{array}{ccccc}
d_{11} & d_{12} & d_{13} & \ldots & d_{1 N_{P}} \\
d_{21} & d_{22} & d_{23} & \ldots & d_{2 N_{P}} \\
\vdots & \vdots & \vdots & \vdots & \vdots \\
d_{N_{V} 1} & d_{N_{V} 2} & d_{N_{V} 3} & \ldots & d_{N_{V} N_{P}}
\end{array}\right]
$$

where $d_{n m} \in \Re$ is the distance between $n$-th EV and charging station deployed at $m$-th location.

The utility function can be defined for the scheduling optimization of $n$-th $\mathrm{EV}$ for charging station deployed at $m$-th location as:

$$
\Delta_{n, m}=u_{n m} \phi\left(d_{n m}\right)+\sum_{g \in G} v_{m}^{g} \psi\left(p_{m}^{g}, t^{g}\right)
$$

where $\phi\left(d_{n m}\right)$ is the cost to travel distance $d_{n m}, v_{m}^{g}$ determines the mode of charging, $p_{m}^{g}$ is the charging price plus parking price, and $t^{g}$ is the number of slots allocated for charging. $\psi\left(p_{m}^{g}, t^{g}\right)$ is the cost which includes price for charging. The utility minimization problem can be formulated as:

$$
\min _{U, v_{m}^{g}}: \sum_{n} \sum_{m} \Delta_{n, m}
$$

Subject to:

$$
\begin{aligned}
C 1: & \sum_{n} u_{n m} \leq 1, \forall m \\
C 2: & \sum_{m} u_{n m} \leq 1, \forall n \\
C 3: & \sum_{g} v_{m}^{g} \leq 1, \forall m \\
C 4: & \sum_{g \in G} v_{m}^{g} \Phi \leq \sum_{n} u_{n m}\left(T_{n}\right), \forall m \\
C 5: & \sum_{n} \sum_{m} u_{n m} \geq \beta N_{v} \\
C 6: & \beta \in[0,1]
\end{aligned}
$$

where charging time should take care of travel time $t_{n m}^{\text {distanceTravel }}$, time demand of the $n$-th $\mathrm{EV} T_{n}$, wait time for the $m$-th station $t_{m}^{\text {wait }}$, and the number of slots allocated to the $n$ - $t h \mathrm{EV} t^{g}$; we collectively denote them $\Phi=\left(t^{g}+\right.$ $\left.t_{m}^{\text {wait }}+t_{n m}^{\text {distanceTravel }}\right)$. The $t_{n m}^{\text {distanceTravel }}, t_{m}^{\text {wait }}$, and $t^{g}$ will be collected at server by having the most updated information from both EVs and charging stations. The constraints 1-5 ensures the following: $\mathrm{C} 1$ : an EV can only be charged at one charging station, C2: a charging station can only serve one $\mathrm{EV}$ at any given time, C3: a charging station can only select one charging mode option at any given time, $\mathrm{C} 4$ : ensures that charging time is within the range of time demand of EVs. C5: the number of EVs served must be greater than $\beta \%$.

Similar to (2), one potential approach is to get an optimal solution to the problem in (4) is to compute all possible combinations of $n$ and $m$. This is not suitable for a solution for a serving area with a large number of EVs and charging stations. Therefore, we consider a branch and bound algorithm for the scheduling of EVs (similar to Algorithm 1). Once the scheduling process is optimized at the server, then an acknowledgment is sent to the EV with information about the selected charging station, charging mode, and the cost. The EVs after getting all the information will send a packet to agree with the given parameters. Finally, a booked message is sent to the charging station, which is selected as an optimal charging station for a given budget and distance to the EV.

\section{Simulation Results}

In this section, we analyze the performance of proposed framework for the optimal placement of charging stations and scheduling optimization of the EVs charging. For the optimal placement of charging infrastructure, we evaluate the performance in terms of normalized values of cost and the number of connectors by considering the number of potential locations $N_{P}=10-20$. We consider two cases based on the number of EVs and $\beta$ (percentage of EVs that should be charged simultaneously): i) $N_{V}=200$ for $\beta=50 \%$ and $80 \%$ and ii) $N_{V}=300$ for $\beta=50 \%$ and $80 \%$. We also consider two cases for scheduling optimization of EVs in smart cities: i) $N_{V}=20 \mathrm{EVs}$ and $N_{P}=40$ charging stations in 


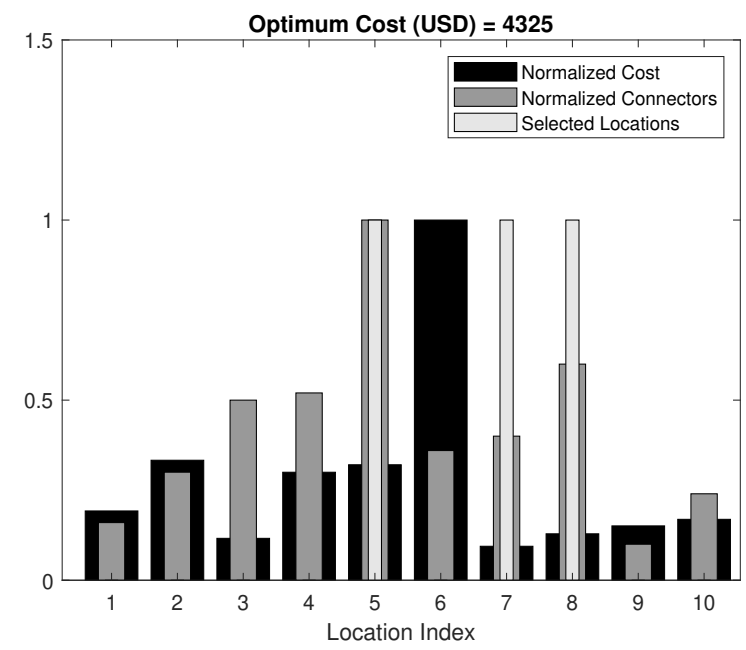

(a)

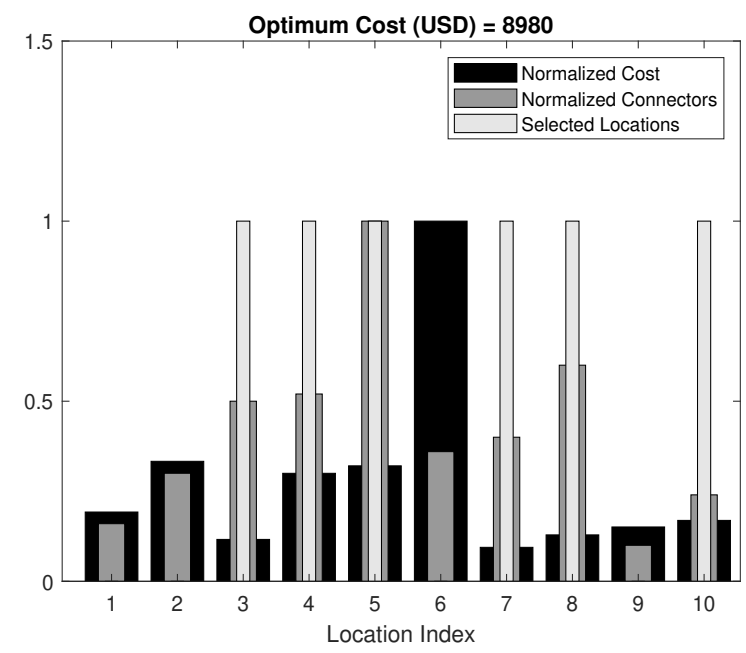

(b)

Fig. 3. Performance evaluation of optimal placement of charging infrastructure: normalized values of the cost and the number of connectors versus location index for individual potential locations for (a) $N_{p}=10, N_{v}=200$, $\beta=50$ and (b) $N_{p}=10, N_{v}=200, \beta=80$.

a rectangular field of $2000 \mathrm{~m} \times 2000 \mathrm{~m}$ for $\beta=50 \%$ and ii) $N_{V}=30 \mathrm{EVs}$ and $N_{P}=40$ charging stations in a rectangular field of $2000 \mathrm{~m} \times 2000 \mathrm{~m}$ for $\beta=100 \%$.

\section{A. Placement of Charging Infrastructure}

Figs. 3(a) and 3(b) depict the normalized values of cost and the number of connectors versus location index for individual potential locations for $\beta=50$ and 80 , respectively. In both cases, the number of potential locations $N_{P}=10$, number of EVs $N_{V}=200$, and the maximum number of connectors $Y_{\max }=50$. The optimally selected locations for charging infrastructure placement are also shown for a given $\beta$ in Figs. 3(a) and 3(b). A $\beta=50$ means, we want at least $50 \%$ vehicles to be charged simultaneously through all selected charging stations. The number of optimally selected locations in case of Fig. 3(a) $(\beta=50 \%)$ is three (location 5,7 , and 8) and the optimum cost is 4,325 USD. In case of Fig. 3(b) $(\beta=80 \%)$ the number of optimally selected locations is six (location 3 , $4,5,7,8$, and 10) and the optimum cost is 8,980 USD. It is evident that the optimum cost for charging infrastructure deployment increase when the value of $\beta$ increases, i.e., more charging infrastructure is required for the higher values of $\beta$.

Similarly, Figures 4(a) and 4(b) show the normalized values of cost and the number of connectors versus location index for individual potential locations for $\beta=50$ and 80 , respectively. However, we consider the number of potential locations $N_{P}=$ $10-20, N_{V}=300$, and $Y_{\max }=50$. The number of optimally selected locations in case of Fig. 4(a) $(\beta=50)$ is five (location $3,4,5,7$, and 8 ) and the optimum cost is 7,635 USD. In case of Fig. 4(b) $\left(N_{P}=20\right.$ and $\left.\beta=80\right)$ the number of optimally selected locations is sixteen and the optimum cost is 18,885 USD. Once again it is clear that the optimum cost for charging infrastructure deployment increase with the increase in $\beta$ and number of EVs in the area $N_{V}$.

\section{B. Scheduling Optimization of EVs Charging}

Fig. 5 shows the randomly distributed EVs $N_{v}=40$ and 20 charging stations deployed using the results of optimization problem in (2). We consider $\beta=50 \%$ (i.e., at least half of the EVs should charge simultaneously by all charging stations). For a given budget and the locations of the EV, the scheduling optimization suggests an appropriate charging station to the EV. It is noted in Fig. 5 that exactly half of the EVs (i.e., 20) are connected with the charging stations. For a given scenario, it is possible that multiple EVs can be connected to a single charging station depending on the number of connectors.

Figs. 6(a) and (b) depict the number of time slots for EV constraint, wait time, and slots allocated at each charging station versus location index for individual charging stations. We consider $N_{p}=20, \beta=50 \%$ and $N_{p}=30, \beta=100 \%$ charging stations for $N_{v}=40$ EVs in Figs. 6(a) and (b) respectively. The allocated slots plus wait time should be in the given constraint for each EV. It can be observed that for some charging stations, EVs have to wait before starting to charge and for others, they can start immediately. It is noted that at all charging stations, the allocated slot time plus wait time is within the given constraint of the EVs. Thus, scheduling optimization can help EV users to find an optimal charging station for their given budget requirements in the IoV-based charging management system. Similarly, it helps charging service providers to know about the demand and budget of EV users, which can certainly help them to improve their infrastructure.

The proposed scheduling scheme may see some unexpected errors in the case of damaged infrastructure, sudden road block because of accidents or similar reasons, electricity blackout, and lack of connectors at charging stations.

\section{COnClusions And Future Directions}

In this paper, we proposed an IoV-based framework for the charging management of EVs. We considered two main aspects of IoV-based charging management: mobile charging infrastructure deployment and scheduling optimization for 


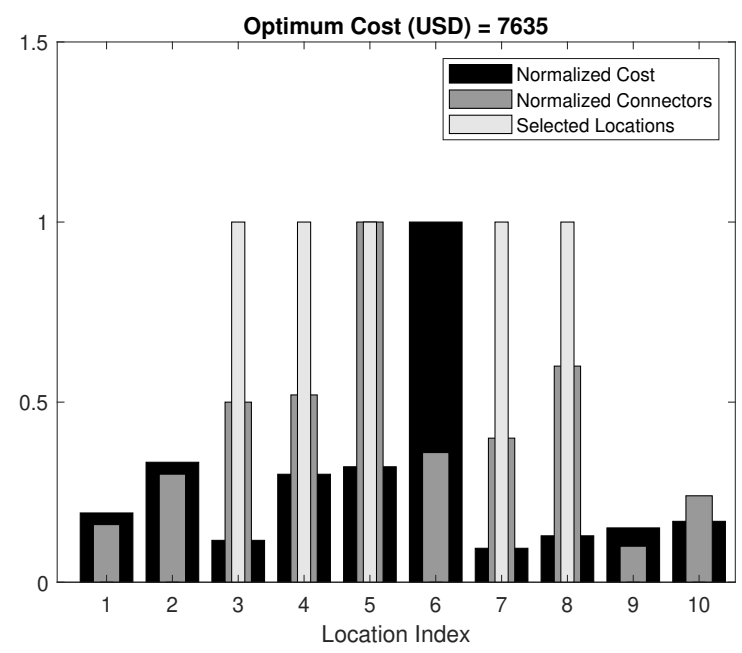

(a)

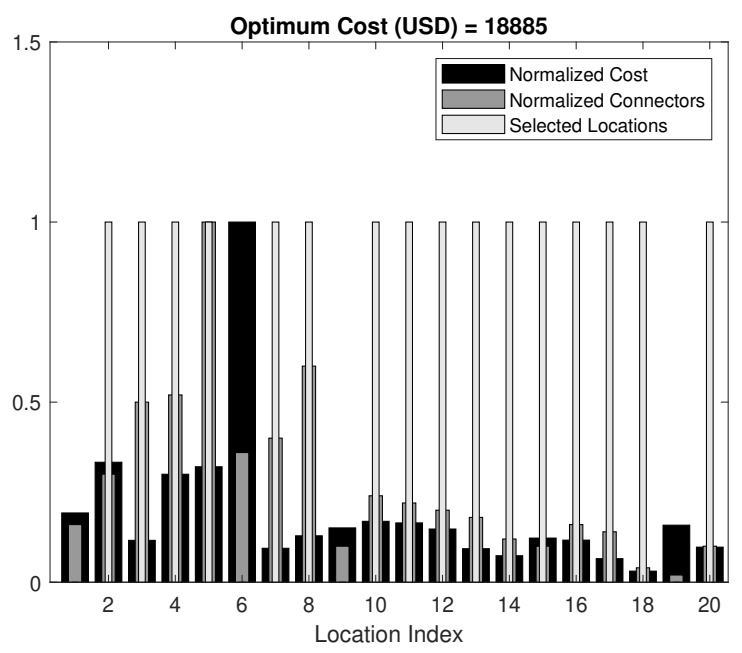

(b)

Fig. 4. Performance evaluation of optimal placement of charging infrastructure: normalized values of the cost and the number of connectors versus location index for individual potential locations for (a) $N_{p}=10, N_{v}=300, \beta=50$, and (b) $N_{p}=20, N_{v}=300, \beta=80$.

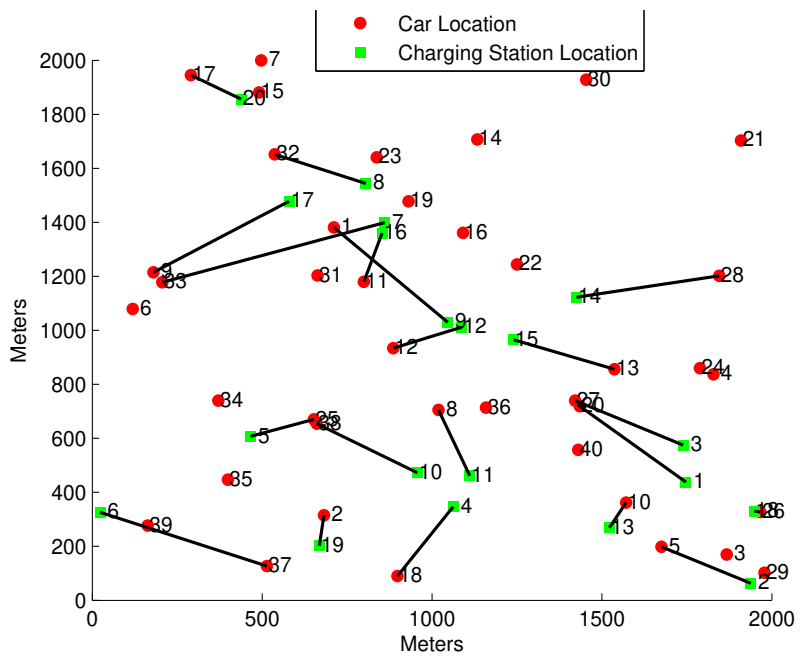

Fig. 5. Optimally assigned charging stations to EVs for 20 deployed charging stations and $40 \mathrm{EVs}$.

EVs. We first formulated an optimization problem for the optimal placement of charging stations to minimize the overall installation cost of charging stations. We then proposed a scheduling optimization of EVs charging to minimize the utility (which consists of the cost to travel, cost of charging, cost to travel to the charging station, and cost for the time EV has to wait before charging). We adopted the branch and bound algorithm to find optimal solutions for the proposed formulations. Extensive simulation results have been presented to support the effectiveness of the proposed framework. It is emphasized that the scheduling of EVs allocates charging stations according to the budget requirements of the EV users and can also reduce the wait time before charging for EV users. The potential limitations of the proposed work are the assumptions used in this paper, i.e., sufficient electricity supply for charging stations and reliable communication infrastructure is available for communication between EVs, charging stations, and server.

Possible future directions for the IoV-enabled efficient energy management are: 1) the proposed scheduling optimization problem can be studied by considering renewable energy resources, such as solar and wind for the charging stations. These charging stations will enable EV users to purchase cheap charging as compared to the utility, 2) mobility of EVs can be incorporated for efficient charging management, 3 ) by using the vehicle to grid (V2G) technology, the peak energy requirement problems can be handled effectively, by selling electricity back to the grid from vehicle battery in peak hours that results in solving peak clipping problem. Similarly, in the non-peak hours, customers can charge their EVs by purchasing cheap electricity, and 4) the proposed solution may have privacy concerns (such as location and behavior tracking) for EVs users, which need to be addressed for the successful deployment of IoV-enabled system.

\section{REFERENCES}

[1] W. Shuai, P. Maillé, and A. Pelov, "Charging electric vehicles in the smart city: a survey of economy-driven approaches," IEEE Transactions on Intelligent Transportation Systems, vol. 17, no. 8, pp. 2089-2106, Aug. 2016.

[2] A. Fachechi, L. Mainetti, L. Palano, L. Patrono, M. Stefanizzi, R. Vergallo, P. Chu, and R. Gadh, "A new vehicle-to-grid system for battery charging exploiting IoT protocols," in IEEE International Conference on Industrial Technology (ICIT), (Seville), pp. 2154-2159, IEEE, Mar. 2015.

[3] S. Anbalagan, D. Kumar, G. Raja, W. Ejaz, A. K. Bashir, et al., "SDN-assisted efficient LTE-WiFi aggregation in next generation IoT networks," Future Generation Computer Systems, vol. 107, pp. 898908, Jun. 2020.

[4] A. Abbas, I. F. Siddiqui, S. U.-J. Lee, A. K. Bashir, W. Ejaz, and N. M. F. Qureshi, "Multi-objective optimum solutions for IoT-based feature models of software product line," IEEE Access, vol. 6, pp. 12228-12239, Feb. 2018

[5] L. Yao, Y.-Q. Chen, and W. H. Lim, "Internet of things for electric vehicle: An improved decentralized charging scheme," in IEEE International Conference on Data Science and Data Intensive Systems, (Sydney, NSW, Australia), pp. 651-658, IEEE, Dec. 2015. 


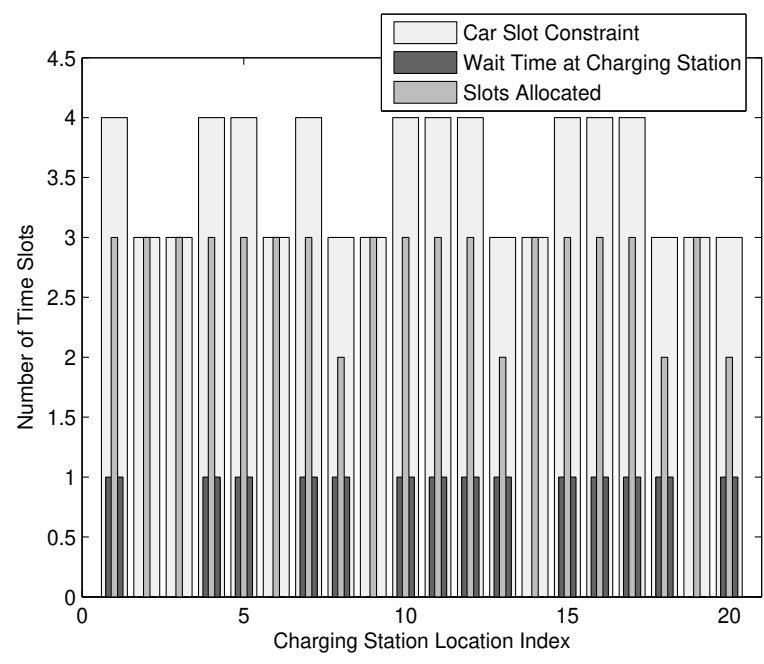

(a)

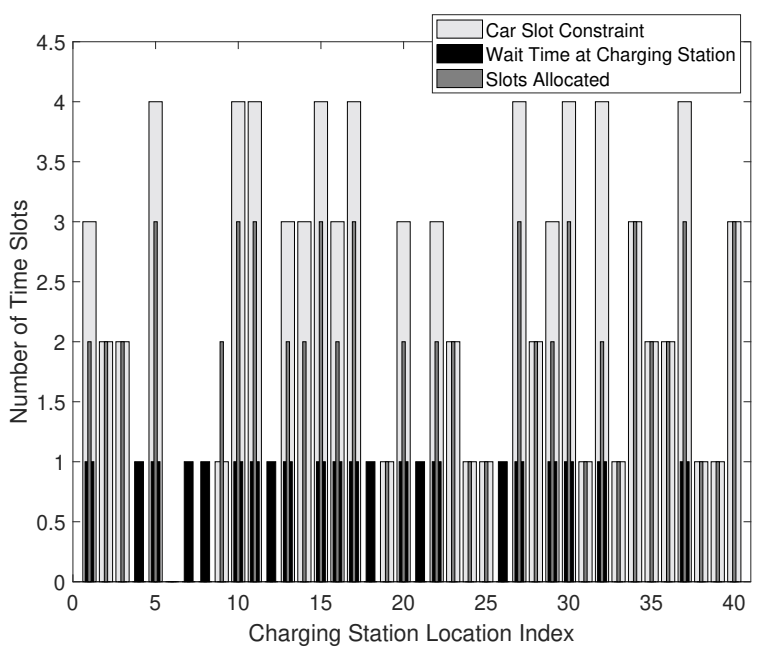

(b)

Fig. 6. Performance evaluation of scheduling optimization in terms of number of time slots for EV constraint, wait time, and slots allocated at each charging station versus location index for individual charging stations for (a) $N_{p}=20 \mathrm{EVs}$ and $N_{v}=40$ charging stations for $\beta=50 \%$ and (b) $N_{p}=30 \mathrm{EVs}$ and $N_{p}=40$ charging stations for $\beta=100 \%$.

[6] H. Chen, Z. Su, Y. Hui, and H. Hui, "Dynamic charging optimization for mobile charging stations in Internet of Things," IEEE Access, vol. 6 , pp. 53509-53520, Sep. 2018.

[7] S. Habib, M. M. Khan, F. Abbas, L. Sang, M. U. Shahid, and H. Tang, "A comprehensive study of implemented international standards, technical challenges, impacts and prospects for electric vehicles," IEEE Access, vol. 6, pp. 13866-13890, Mar. 2018.

[8] Y. He, Z. Song, and Z. Liu, "Fast-charging station deployment for battery electric bus systems considering electricity demand charges," Sustainable Cities and Society, vol. 48, p. 101530, Jul. 2019.

[9] X. Wang, C. Sun, R. Wang, and T. Wei, "Two-stage optimal scheduling strategy for large-scale electric vehicles," IEEE Access, vol. 8, pp. 13821-13832, Jan. 2020.

[10] M. H. Amini, M. P. Moghaddam, and O. Karabasoglu, "Simultaneous allocation of electric vehicles' parking lots and distributed renewable resources in smart power distribution networks," Sustainable Cities and Society, vol. 28, pp. 332-342, Jan. 2017.

[11] M. Xu and Q. Meng, "Optimal deployment of charging stations considering path deviation and nonlinear elastic demand," Transportation Research Part B: Methodological, vol. 135, pp. 120-142, May 2020.

[12] Z. Luo, F. He, X. Lin, J. Wu, and M. Li, "Joint deployment of charging stations and photovoltaic power plants for electric vehicles," Transportation Research Part D: Transport and Environment, vol. 79, p. 102247 , Feb. 2020

[13] M. Campaña and E. Inga, "Heuristic method for optimal deployment of electric vehicle charge stations using linear programming," in IEEE Colombian Conference on Applications in Computational Intelligence, pp. 247-258, Springer, Dec. 2019.

[14] Q. Cui, Y. Weng, and C.-W. Tan, "Electric vehicle charging station placement method for urban areas," IEEE Transactions on Smart Grid, vol. 10, no. 6, pp. 6552-6565, Nov. 2019.

[15] D. Gong, M. Tang, B. Buchmeister, and H. Zhang, "Solving Location Problem for Electric Vehicle Charging StationsâA Sharing Charging Model," IEEE Access, vol. 7, pp. 138391-138402, Sep. 2019.

[16] M. M. Islam, H. Shareef, and A. Mohamed, "Optimal location and sizing of fast charging stations for electric vehicles by incorporating traffic and power networks," IET Intelligent Transport Systems, vol. 12, no. 8, pp. 947-957, Oct. 2018.

[17] K. C. Arayici and G. Poyrazoglu, "The optimal placement model for electric vehicle charging stations," in 2019 International Conference on Power Generation Systems and Renewable Energy Technologies (PGSRET), pp. 1-4, IEEE, 2019.

[18] H. Ahmadi-Nezamabad, M. Zand, A. Alizadeh, M. Vosoogh, and S. Nojavan, "Multi-objective optimization based robust scheduling of electric vehicles aggregator," Sustainable Cities and Society, vol. 47, p. 101494, May 2019.
[19] E. Yao, T. Liu, T. Lu, and Y. Yang, "Optimization of electric vehicle scheduling with multiple vehicle types in public transport," Sustainable Cities and Society, vol. 52, p. 101862, 2020.

[20] W. Tang and Y. J. A. Zhang, "A model predictive control approach for low-complexity electric vehicle charging scheduling: Optimality and scalability," IEEE Transactions on Power Systems, vol. 32, no. 2, pp. 1050-1063, Mar. 2017.

[21] X. Tang, S. Bi, and Y.-J. A. Zhang, "Distributed routing and charging scheduling optimization for Internet of electric vehicles," IEEE Interne of Things Journal, vol. 6, no. 1, pp. 136-148, Feb. 2019.

[22] E. Ucer, I. Koyuncu, M. C. Kisacikoglu, M. Yavuz, A. Meintz, and C. Rames, "Modeling and analysis of a fast charging station and evaluation of service quality for electric vehicles," IEEE Transactions on Transportation Electrification, vol. 5, no. 1, pp. 215-225, Mar. 2019.

[23] D. Kosmanos, L. A. Maglaras, M. Mavrovouniotis, S. Moschoyiannis, A. Argyriou, A. Maglaras, and H. Janicke, "Route optimization of electric vehicles based on dynamic wireless charging," IEEE Access, vol. 6, pp. 42551-42565, Jul. 2018.

[24] Y. Cao, L. Huang, Y. Li, K. Jermsittiparsert, H. Ahmadi-Nezamabad, and S. Nojavan, "Optimal scheduling of electric vehicles aggregator under market price uncertainty using robust optimization technique," International Journal of Electrical Power \& Energy Systems, vol. 117, p. 105628, May 2020.

[25] S. S. Barhagh, M. Abapour, and B. Mohammadi-Ivatloo, "Optimal scheduling of electric vehicles and photovoltaic systems in residential complexes under real-time pricing mechanism," Journal of Cleaner Production, vol. 246, p. 119041, Feb. 2020.

[26] Y. Zhang, P. You, and L. Cai, "Optimal charging scheduling by pricing for EV charging station with dual charging modes," IEEE Transactions on Intelligent Transportation Systems, vol. 20, no. 9, pp. 3386-3396, Sep. 2019.

[27] K. L. López, C. Gagné, and M.-A. Gardner, "Demand-side management using deep learning for smart charging of electric vehicles," IEEE Transactions on Smart Grid, vol. 10, no. 3, pp. 2683-2691, May 2019.

[28] X. Tan, G. Qu, B. Sun, N. Li, and D. H. Tsang, "Optimal scheduling of battery charging station serving electric vehicles based on battery swapping," IEEE Transactions on Smart Grid, vol. 10, no. 2, pp. 13721384, Mar. 2019.

[29] A. Hess, F. Malandrino, M. B. Reinhardt, C. Casetti, K. A. Hummel, and J. M. Barceló-Ordinas, "Optimal deployment of charging stations for electric vehicular networks," in Proceedings of the first workshop on Urban networking, (Nice, France), pp. 1-6, ACM, Dec. 2012.

[30] S. H. Committee et al., "SAE charging configurations and ratings terminology," Society of Automotive Engineers, 2011.

[31] N. Thakoor and J. Gao, "Branch-and-bound for model selection and its computational complexity," IEEE Transactions on Knowledge and Data Engineering, vol. 23, no. 5, pp. 655-668, May 2011. 
[32] M. Y. Naderi, P. Nintanavongsa, and K. R. Chowdhury, "RF-MAC: A medium access control protocol for re-chargeable sensor networks powered by wireless energy harvesting," IEEE Transactions on Wireless Communications, vol. 13, no. 7, pp. 3926-3937, Jul. 2014.

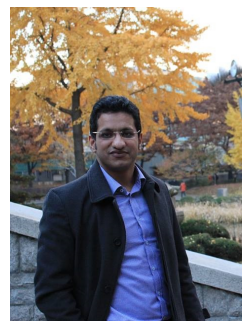

Waleed Ejaz (Sâ12-Mâ14-SMâ16) is an Assistant Professor in the Department of Applied Science Engineering at Thompson Rivers University, Kamloops, BC, Canada. Previously, he held academic and research positions at Ryerson University, Carleton University, and Queenâs University in Canada. He received the B.Sc. and M.Sc. degrees in Computer Engineering from the University of Engineering and Technology, Taxila, Pakistan and the National University of Sciences and Technology, Islamabad, Pakistan, and the Ph.D. degree in Information and Communication Engineering from Sejong University, Republic of Korea, in 2014. He has co-authored over 90 papers in prestigious journals and conferences, and 3 books. His current research interests include Internet of Things (IoT), energy harvesting, 5G and beyond networks, and mobile edge computing. He is an Associate Editor of the IEEE Communications Magazine, IEEE Canadian Journal of Electrical and Computer Engineering, and the IEEE ACCESS. Dr. Ejaz completed certificate courses on âTeaching and Learning in Higher Educationâ from the Chang School at Ryerson University. He is a registered Professional Engineer (P.Eng.) in the province of British Columbia, Canada. Dr. Ejaz is a senior member of IEEE, member of ACM, and ACM distinguished speaker.

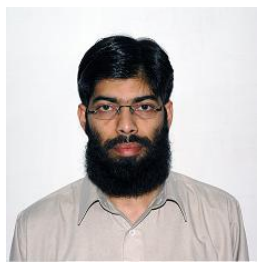

Muhammad Naeem received the BS (2000) and MS (2005) degrees in Electrical Engineering from the University of Engineering and Technology, Taxila, Pakistan. He received his $\mathrm{PhD}$ degree (2011) from Simon Fraser University, BC, Canada. From 2012 to 2013, he was a Postdoctoral Research Associate with WINCORE Lab. at Ryerson University, Toronto, ON, Canada. Since August 2013, he has been an assistant professor with the Department of Electrical Engineering, COMSATS Institute of IT, Wah Campus, Pakistan and Research Associate with WINCORE Lab. at Ryerson University. From 2000 to 2005, he was a senior design engineer at Comcept (pvt) Ltd. At the design department of Comcept (pvt) Ltd, Dr. Naeem participated in the design and development of smart card based GSM and CDMA pay phones. Dr. Naeem is also a Microsoft Certified Solution Developer (MCSD). His research interests include optimization of wireless communication systems, non-convex optimization, resource allocation in cognitive radio networks and approximation algorithms for mixed integer programming in communication systems. Dr. Naeem has been the recipient of NSERC CGS scholarship.

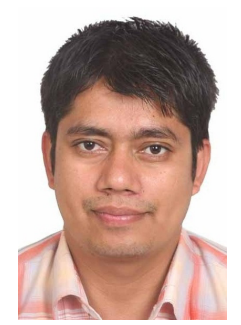

Shree Krishna Sharma (S'12-M'15-SM'18) is currently Research scientist at the Interdisciplinary Center for Security, Reliability and Trust (SnT), University of Luxembourg. Prior to this, he held research positions at the University of Western Ontario, Canada, and Ryerson University, Canada; and also worked as a Research Associate at the SnT after receiving his $\mathrm{PhD}$ degree in Wireless Communications from the University of Luxembourg in 2014. $\mathrm{He}$ has published more than 100 technical papers in scholarly journals, international conferences, and book chapters, and has over 2400 google scholar citations with an h-index of 24. His current research interests include $5 \mathrm{G}$ and beyond wireless, Internet of Things, machine-type communications, machine learning, edge computing and optimization of distributed communications, computing and caching resources. $\mathrm{He}$ is a Senior Member of IEEE and is the recipient of several prestigious awards including âFNR Award for Outstanding PhD Thesis 2015â from FNR, Luxembourg, âBest Paper Awardâ in CROWNCOM 2015 conference, â2018 EURASIP JWCN Best Paper Awardâ and the co-recipient of âFNR Award for Outstanding Scientific Publication 2019â.

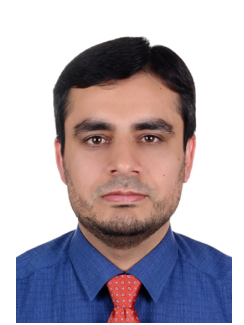

Asad Masood Khattak is an Associate Professor at the College of Technological Innovation, Zayed University in Abu Dhabi, UAE that he joined in August 2014. He received his M.S. in Information Technology from National University of Sciences and Technology (NUST), Islamabad, Pakistan in 2009 and received his Ph.D. degree in Computer Engineering from Kyung Hee University, South Korea in 2012. He worked as Post-Doctoral Fellow at Department of Computer Engineering, Kyung Hee University, South Korea and later joined the same college as Assistant Professor. He is currently leading three research projects, collaborating in four research projects, and has successfully completed five research projects in the fields of Data Curation, Context-aware Computing, IoT, and Secure Computing. He is IEEE member and has authored/coauthored more than 120 journal and conference articles in highly reputed venues. He is serving as reviewer, program committee member, organizer, and guest editor of many workshops, conferences, and journals. He has delivered keynote speeches, invited talks, guest lectures and has delivered short courses in many universities. He and his team have secured several national and international awards in different competitions.

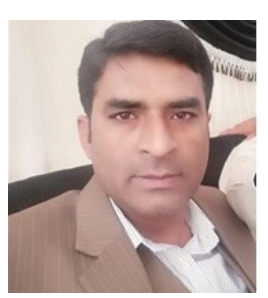

Muhammad Rashid Ramzan received the MS in Electrical Engineering from Department of electrical and computer engineering, Comsats University Islamabad, Wah campus. He is currently working towards his $\mathrm{PhD}$ degree from the same department. His current research interests include the topics related to applied optimization, targeting telecommunication and smart system applications.

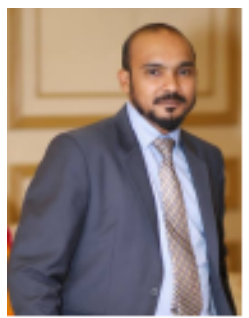

Amjad Ali received his B.S. and M.S. degrees in Computer Science from COMSATS Institute of Information Technology, Pakistan in 2006 and 2008, respectively. He received his $\mathrm{PhD}$ in Electronics and Radio Engineering from Kyung Hee University, South Korea in 2015. Since 2015, he is serving as Assistant Professor at the Department of Computer Science, COMSATS University Islamabad, Lahore Campus, Pakistan. From Feb. 2018 to March 2019, he was the Postdoctoral scholar with the UWB Wireless Communications Research Center (formerly Key National IT Research Center) at the Department of Information and Communication Engineering, Inha university, South Korea and with the Mobile Network and Communications Lab at the School of Electrical Engineering, Korea University, Seoul, South Korea. His main research interests include Cognitive Radio Networks, Multimedia Communications, Cloud Computing, Machine Learning, Internet of Things, and Vehicular Networks.

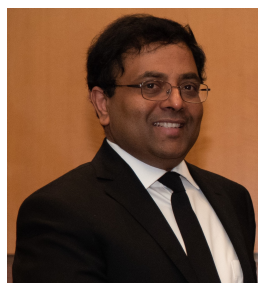

Alagan Anpalagan (S98-M01-SM04) is a Professor in the ELCE Department, Ryerson University, Canada. He served the department in administrative positions as Associate Chair, Program Director for Electrical Engineering, and Graduate Program Director. He directs a research group working on radio resource management, and radio access and networking areas within the WINCORE Lab. He has coauthored four edited books and two books in wireless communication and networking areas. He serves as Vice Chair, IEEE SIG on Green and Sustainable Networking and Computing with Cognition and Cooperation. Dr. Anpalagan was the recipient of the IEEE Canada J.M. Ham Outstanding Engineering Educator Award (2018), SGS Outstanding Contribution to Graduate Education Award (2017), Deans Teaching Award (2011), Faculty Scholastic, Research and Creativity Awards from the Ryerson University. He was also the recipient of IEEE M.B. Broughton Central Canada Service Award (2016), Exemplary Editor Award from IEEE ComSoc (2013) and, a co-author of a paper that received IEEE SPS Young Author Best Paper Award (2015). He is a Registered Professional Engineer in the province of Ontario, Canada and Fellow of the Institution of Engineering and Technology (FIET) and Fellow of the Engineering Institute of Canada (FEIC). 